Marta Lewicka · Marco Spadini

\title{
A remark on the genericity of multiplicity results for forced oscillations on manifolds
}

Received: January 24, 2000; in final form: January 16, 2001

Published online: March 19, 2002 - (C) Springer-Verlag 2002

\begin{abstract}
We discuss the genericity of some multiplicity results for periodically perturbed autonomous first- and second-order ODEs on manifolds.

In particular, the genericity of the following property is investigated: if the differentiable manifold $M$ is compact, then the equation $\ddot{x}_{\pi}=h(x, \dot{x})+f(t, x, \dot{x})$ on $M$ has $|\chi(M)|$ geometrically distinct $T$-periodic solutions for any small enough $T$-periodic perturbing function $f$.
\end{abstract}

Mathematics Subject Classification (2000). 34C25, 37C27

\section{Introduction}

Let $M \subset \mathbb{R}^{k}$ be a boundaryless smooth manifold. In our recent work [6] the genericity of the following property has been proved: if M is compact, the perturbed autonomous equation on $M$

$$
\ddot{x}_{\pi}=g(x)+f(t, x, \dot{x})
$$

has $|\chi(M)|$ geometrically distinct $T$-periodic solutions for any 'small' perturbation $f$ that is $T$-periodic in $t$.

In this paper, which can be seen as a continuation of our research in [6], we want to discuss the same property, relatively to the following equation (Theorem 4.4):

$$
\ddot{x}_{\pi}=h(x, \dot{x})+f(t, x, \dot{x}),
$$

where $h: T M \longrightarrow \mathbb{R}^{k}$ is $C^{r}$ and tangent to $M$, and the perturbing function $f: \mathbb{R} \times T M \longrightarrow \mathbb{R}^{k}$ is $T$-periodic in $t$ (with $T>0$ a fixed number), tangent to $M$ and satisfies the usual Carathéodory and admissibility conditions.

In particular, we shall prove that when $M$ is compact, then the set of $h$ such that (2) admits at least $|\chi(M)|$ geometrically distinct $T$-periodic solutions for $f$ small enough, is open and dense in the set of all the $C^{r}$ tangent vector fields (Corollary 4.5).

M. Lewicka: Max-Planck Institute for Mathematics in the Sciences, Inselstrasse 22-26, 04103 Leipzig, Germany

M. Spadini: Dipartimento di Matematica Applicata "G. Sansone”, Facoltà di Ingegneria, Via S. Marta 3, 50139 Firenze, Italy 
The genericity result relative to (2) does not seem to be attainable directly with the methods of [6]. In fact, we proceed in two steps: first, we obtain results in the spirit of [6] but for first-order equations in the non-compact case. Secondly, using the fact that every second-order ODE on $M$ is equivalent to a suitable first-order equation on the tangent bundle $T M$, we get a genericity result for second-order equations on (not necessarily compact) manifolds (Theorem 4.4) that reduces to the quoted result when $M$ is compact.

In the following, we use the same terminology of [6], and refer to [5,8] for the notions of differential topology.

\section{Preliminaries and notation}

Let $N \subset \mathbb{R}^{l}$ be a boundaryless, $n$-dimensional, smooth manifold. The general form of the first-order ODE on $N$ studied here is the following:

$$
\dot{x}=\varphi(x)+\gamma(t, x)
$$

where $\varphi: N \longrightarrow \mathbb{R}^{l}$ is $C^{r}$, tangent to $N$ and admissible, i.e. such that $\varphi^{-1}(0)$ is compact. The perturbation $\gamma: \mathbb{R} \times N \longrightarrow \mathbb{R}^{l}$ is assumed to have the following properties:

(P1) (Carathéodory, $T$-periodicity in $t$ )

- for any $p \in N, \gamma(\cdot, p): \mathbb{R} \longrightarrow \mathbb{R}^{l}$ is measurable and $T$-periodic,

- for a.a. $t \in \mathbb{R}, \gamma(t, \cdot): N \longrightarrow \mathbb{R}^{l}$ is continuous;

(P2) (tangency)

- for any $p \in N$ and for a.a. $t \in \mathbb{R}, \gamma(t, p) \in T_{p} N$;

(P3) (admissibility)

- for any compact $K \subset N$ there exists a function $h_{K} \in L^{1}([0, T], \mathbb{R})$ such that for a.a. $t \in[0, T]$, for any $p \in K$,

$$
|\gamma(t, p)|<h_{K}(t) .
$$

By $T M$ we mean the tangent bundle to the embedded manifold $M$, that is the subset of $\mathbb{R}^{k} \times \mathbb{R}^{k}$ given by

$$
T M=\left\{(p, v) \in \mathbb{R}^{k} \times \mathbb{R}^{k}: p \in M, v \in T_{p} M\right\} .
$$

We will say that a continuous map $\varphi: \mathbb{R} \times T M \rightarrow \mathbb{R}^{k}$ is tangent to $M$ provided that $\varphi(t, q, v) \in T_{q} M$ for all $(t, q, v) \in \mathbb{R} \times T M$.

In what follows, the symbol $C_{T}^{1}(M)$ will denote the metric subspace of the Banach space $\left(C_{T}^{1}\left(\mathbb{R}^{k}\right),\|\cdot\|_{1}\right)$ of all the $T$-periodic, $C^{1}$ functions $x: \mathbb{R} \longrightarrow M$ with the usual $C^{1}$ norm $\|\cdot\|_{1}$. Analogously, by $C_{T}(T M)$ we mean the metric space of $T$-periodic, continuous functions $x: \mathbb{R} \longrightarrow T M$, with the metric inherited from the Banach space $C_{T}\left(\mathbb{R}^{k} \times \mathbb{R}^{k}\right)$.

As in [4], we tacitly assume some natural identifications; for example, we identify a point $p \in M$ with the constant function $t \mapsto p$ in $C_{T}^{1}(M)$, or a function $x \in C_{T}^{1}(M)$ with $(x, \dot{x}) \in C_{T}(T M)$. Moreover, $M$ is regarded as the zero section of 
$T M$, so that, given $h: T M \rightarrow \mathbb{R}^{k}$, by $\left.h\right|_{M}: M \longrightarrow \mathbb{R}^{k}$ we understand the function $\left.h\right|_{M}(p)=h(p, 0)$.

Recall that $x$ is a solution of (2) if $\dot{x}$ is absolutely continuous, and for a.a. $t \in \mathbb{R}$

$$
\ddot{x}_{\pi}(t)=h(x(t), \dot{x}(t))+f(t, x(t), \dot{x}(t)),
$$

where $\ddot{x}_{\pi}(t)$ is the orthogonal projection of $\mathbb{R}^{k}$ onto $T_{x(t)} M$.

Equation (2) is equivalent to the following ODE on TM:

$$
\dot{\xi}=\widehat{h}(\xi)+\bar{f}(t, \xi),
$$

where, given $\xi=(p, v)$ with $p \in M$ and $v \in T_{p} M, \widehat{h}(p, v)=(v, r(p, v)+h(p, v))$ and $\bar{f}(t, p, v)=(0, f(t, p, v))$. The above map $r: T M \longrightarrow \mathbb{R}^{k}$ assigns to any fixed $(q, v) \in T M$ the unique vector in $\mathbb{R}^{k}$ which makes $(v, r(q, v))$ tangent to $T M$ at $(q, v)$. It is known that $r(q, v) \in T_{q} M^{\perp}$. In this way $\widehat{h}$ (as well as $\bar{f}$ ) is tangent to $T M$. In the following, given $h: T M \longrightarrow \mathbb{R}^{k}$ as above, we will often make use of the associated vector field $\widehat{h}$, which will be referred to as the second-order vector field associated to $h$.

Consider Equation (3). We say that a point $p \in \varphi^{-1}(0) \subset N$ is $T$-resonant for $\varphi$ if (see e.g. [3]):

- $\varphi$ is $C^{1}$ in a neighbourhood of $p$,

- the linearized equation on $T_{p} N$ (note that $\varphi^{\prime}(p) \in \operatorname{End}\left(T_{p} N\right)$ )

$$
\dot{x}=\varphi^{\prime}(p) x
$$

admits nontrivial (i.e. nonzero) $T$-periodic solutions.

Note that $p$ is non- $T$-resonant for $\varphi$ if and only if the spectrum $\operatorname{spec}\left(\varphi^{\prime}(p)\right)$ of $\varphi^{\prime}(p)$ contains no eigenvalues of the form $\frac{2 \pi n i}{T}, n \in \mathbb{Z}$.

Following [6], we say that a point $p \in\left(\left.h\right|_{M}\right)^{-1}(0) \subset M$ is second-order $T$-resonant for $h$, if $(p, 0) \in T M$ is $T$-resonant for $\widehat{h}$. In particular, if $h$ is $C^{1}$ in a neighbourhood of $(p, 0)$ in $T M$ and $D_{2} h(p, 0)=0$, the second-order $T$-resonancy is equivalent to

$$
-\left(\frac{2 n \pi}{T}\right)^{2} \in \operatorname{spec}\left(\left.h\right|_{M}\right)^{\prime}(p) \text { for some } n \in \mathbb{Z} .
$$

As in [6], we denote by $\mathcal{F}(N)$ the topological vector space of all the functions $\gamma: \mathbb{R} \times N \longrightarrow \mathbb{R}^{l}$ having the properties (P1)-(P3), endowed with the topology given by the following fundamental system of neighbourhoods of 0 :

$$
\left\{U_{K, \varepsilon}: K \text { is a compact subset of } N, \varepsilon>0\right\} \text {, }
$$

with

$$
U_{K, \varepsilon}=\{\gamma \in \mathcal{F}(N) \text { : for a.a. } t \in[0, T] \text {, for all } p \in K,|\gamma(t, p)|<\varepsilon\} .
$$

Furthermore, by $\mathscr{E}(M)$ we mean the topological vector space of all the functions $f: \mathbb{R} \times T M \longrightarrow \mathbb{R}^{k}$ with the properties as in Sect. 1, and with the topology inherited from $\mathcal{F}(T M) \supset \mathscr{E}(M)$. 


\section{Genericity of the multiplicity results for first-order equations}

Consider the set $\mathfrak{X}^{r, s}(N), r, s \in \mathbb{N} \cup\{0\}$, of the admissible $C^{r}$ vector fields $\varphi$, tangent to $N$, and such that $|\operatorname{deg}(\varphi, N)|=s$, and let $\mathfrak{X}_{T}^{r, s}(N)$ be its subset determined by the additional condition that (3) has at least $s$ geometrically distinct $T$-periodic solutions for any $\gamma$ in a suitably 'small' neighbourhood of 0 in $\mathcal{F}(N)$. In this section, that is devoted to first-order ODEs on (not necessarily compact) boundaryless manifolds, we show that $\mathfrak{X}_{T}^{r, s}(N)$ is open and dense (with an appropriate topology) in $\mathfrak{X}^{r, s}(N)$.

Let $\mathfrak{X}^{r}(N), r \geq 0$, be the vector space of the $C^{r}$ tangent vector fields to $N$ endowed with the fine (Whitney) topology [5]. For the purpose of future reference, we recall that, given $\varphi \in \mathfrak{X}^{r}(N)$, the basis of its open neighbourhoods consists of the sets

$$
\begin{array}{r}
\mathcal{N}^{r}(\varphi, \Psi, \mathcal{K}, E)=\left\{\omega \in \mathfrak{X}^{r}(N):\left\|D^{k}\left(\varphi \psi_{i}^{-1}\right)(p)-D^{k}\left(\omega \psi_{i}^{-1}\right)(p)\right\|<\varepsilon_{i},\right. \\
\text { for all } \left.p \in \psi_{i}\left(K_{i}\right),|k|=0, \ldots, r, i \in \Lambda\right\},
\end{array}
$$

where $\Psi=\left\{\psi_{i}, U_{i}\right\}_{i \in \Lambda}$ is a locally finite set of charts on $N$, indexed by a set $\Lambda$, $\mathcal{K}=\left\{K_{i}\right\}_{i \in \Lambda}$ is a family of compact subsets $K_{i} \subset U_{i}$, and $E=\left\{\varepsilon_{i}\right\}_{i \in \Lambda}$ a family of positive numbers.

Let $\mathfrak{X}_{a}^{r}(N)$ be the subset of $\mathfrak{X}^{r}(N)$ made up of the $C^{r}$ admissible vector fields. Observe that $\mathfrak{X}_{a}^{r}$ is open whereas, in general, it is not a vector space.

We will say that $s \in \mathbb{N} \cup\{0\}$ is admissible if there exists $\varphi \in \mathfrak{X}_{a}^{r}(N)$ such that $|\operatorname{deg}(\varphi, N)|=s$. Given an admissible $s$, we denote by $\mathfrak{X}^{r, s}(N)$ the set of admissible vector fields $\varphi \in \mathfrak{X}_{a}^{r}(N)$ such that $|\operatorname{deg}(\varphi, N)|=s$. Obviously $\mathfrak{X}^{r, n}(N)$ is not a vector space unless $N$ is compact. In fact, as a consequence of the Poincaré-Hopf theorem, when $N$ is compact, $s=|\chi(N)|$ is the only possible admissible number.

In the following, unless stated differently, $s$ will always denote an admissible integer.

Proposition 3.1. The set $\mathfrak{X}^{r, s}(N), r \geq 0$, is open in $\mathfrak{X}^{r}(N)$.

Proof. Fix a vector field $\varphi \in \mathfrak{X}^{r, s}(N)$. Let $\Psi=\left\{\psi_{i}, U_{i}\right\}_{i \in \Lambda}$ be a locally finite atlas on $N$. Refining $\Psi$ if necessary, we can assume that $\bar{U}_{i}$ is compact for any $i \in \Lambda$. Let $\left\{v_{i}\right\}_{i \in \Lambda}$ be a partition of unity subordinated to the open covering $\left\{U_{i}\right\}_{i \in \Lambda}$ of $N$. For any $i \in \Lambda$, put $K_{i}=\operatorname{supp} v_{i}$. The family of compact subsets $\mathcal{K}=\left\{K_{i}\right\}_{i \in \Lambda}$ is a neighbourhood-finite covering of $N$. Consequently, since $\varphi$ is admissible, there exists a finite set of indices $\left\{i_{1}, \ldots, i_{\sigma}\right\} \subset \Lambda$ such that $\varphi^{-1}(0) \subset \bigcup_{j=1}^{\sigma} U_{i_{j}}$.

Let $E=\left\{\varepsilon_{i}\right\}_{i \in \Lambda}$ be a family of positive numbers. One sees that if for $i \notin$ $\left\{i_{1}, \ldots, i_{\sigma}\right\}$ the $\varepsilon_{i}$ 's are small enough, then

$$
\mathcal{N}^{r}(\varphi, \Psi, \mathcal{K}, E) \subset \mathfrak{X}_{a}^{r}(N) .
$$

By the homotopy property of the degree, assuming $\varepsilon_{i_{j}}$ small enough for $j=$ $1, \ldots, \sigma$, one gets

$$
\mathcal{N}^{r}(\varphi, \Psi, \mathcal{K}, E) \subset \mathfrak{X}^{r, s}(N),
$$

which completes the proof. 
Remark. Note that Proposition 3.1 is false if the Whitney topology is replaced by the compact-open (in $C^{r}$ ) one.

Proposition 3.2. Any open set of $\mathfrak{X}^{r, s}(N), r \geq 0$, contains a vector field $\omega$ whose zeros are all non-degenerate. Consequently, by the additivity of the degree, $\# \omega^{-1}(0) \geq s$.

Proof. We recall that by the Thom transversality theorem, in case $r \geq 1$, the set of the $C^{r}$ tangent vector fields on $N$ whose zeros are non-degenerate is dense in $\mathfrak{X}^{r}(N)$ [5,9]. Since $\mathfrak{X}^{r, s}(N)$ is open in $\mathfrak{X}^{r}(N), U$ is open in $\mathfrak{X}^{r}(N)$.

In the case $r=0$ it is enough to note that $\mathfrak{X}^{1}(N)$ is dense in $\mathfrak{X}^{0}(N)$ and use the argument above.

Lemma 3.3. Assume that $\varphi \in \mathfrak{X}^{r, s}(N), r \geq 1$, has $\sigma$ non-degenerate zeros $p_{1}, \ldots$, $p_{\sigma}$. Then, given a neighbourhood $U$ of $\varphi$ in $\mathfrak{X}^{r, s}(N)$, there exists $\omega \in U$ such that $p_{1}, \ldots, p_{\sigma}$ are non-T-resonant zeros of $\omega$.

Proof. For $j=1, \ldots, \sigma$ define a smooth function $w_{j}: N \longrightarrow \mathbb{R}$ by

$$
w_{j}(p)=\frac{1}{2} \eta_{j}(p)\left\|p-p_{j}\right\|^{2}
$$

where $\eta_{j}: N \longrightarrow[0,1]$ is smooth with compact support and is equal to 1 in a neighbourhood of $p_{j}$. Note that $\operatorname{supp} w_{j} \subset \operatorname{supp} \eta_{j}$ and

$$
\left(\varphi+\rho \operatorname{grad} w_{j}\right)^{\prime}\left(p_{j}\right)=\varphi^{\prime}\left(p_{j}\right)+\rho \operatorname{Id}_{T_{p} N} .
$$

Without loss of generality, one can assume that supp $\eta_{k} \cap \operatorname{supp} \eta_{j}=\emptyset$ for $k \neq j$, and $k, j \in\{1, \ldots, \sigma\}$. Define

$$
w=\sum_{j=1}^{\sigma} w_{j}
$$

Take $\omega=\varphi+\rho \operatorname{grad} w$. For $j=1, \ldots, \sigma$ and $\rho>0$ small enough, the spectrum of $\omega^{\prime}\left(p_{j}\right)$ does not contain elements of the form $2 \pi n i / 2, n \in \mathbb{Z}$. From Proposition 3.1 it follows that $\omega$ is in $\mathfrak{X}^{r, s}$ when $\rho$ is small. This proves the assertion.

Denote by $\mathfrak{X}_{T}^{r, s}(N)$ the set consisting of those vector fields $\varphi \in \mathfrak{X}^{r, s}(N)$ for which there exists an open neighbourhood of $U_{\varphi}$ of 0 in $\mathcal{F}(N)$ with the property that Equation (3) admits at least $s$ geometrically distinct $T$-periodic solutions whenever $\gamma$ is taken in $U_{\varphi}$. Our main result states that such a set is generic within $\mathfrak{X}^{r, s}(N)$.

Theorem 3.4. The set $\mathfrak{X}_{T}^{r, s}(N), r \geq 0$, is open in $\mathfrak{X}^{r}(N)$ and dense in $\mathfrak{X}^{r, s}(N)$.

Proof. To prove the first assertion, take $\varphi \in \mathfrak{X}_{T}^{r, s}(N)$ and let $U_{K, \varepsilon} \subset \mathcal{F}(N)$ be such that (3) admits at least $s$ geometrically distinct $T$-periodic solutions whenever 
$\gamma \in U_{K, \varepsilon}$. By Proposition 3.1, take $\mathcal{N}^{r}(\varphi, \Psi, \mathcal{K}, E) \subset \mathfrak{X}^{r, s}(N)$. Obviously, if $\varepsilon_{i}<\varepsilon / 2$ for all $i \in \Lambda$ such that $K_{i} \cap K \neq \emptyset$, then $\mathcal{N}^{r}(\varphi, \Psi, \mathcal{K}, E) \subset \mathfrak{X}_{T}^{r, s}(N)$.

We now prove the density. Since $\mathfrak{X}^{1}(N)$ is dense in $\mathfrak{X}^{0}(N)$, without loss of generality we can assume that $r \geq 1$.

Fix an open subset $U$ of $\mathfrak{X}^{r, s}(N)$. By Proposition 3.2 and Lemma 3.3, there exists $\omega \in U$ with at least $s$ non- $T$-resonant zeros $p_{1}, \ldots, p_{s}$. Let us prove that $\omega \in \mathfrak{X}_{T}^{r, s}(N)$. Indeed, the proof of Theorem 4.1 in [6] shows that for every $p_{i}$, $i=1, \ldots, s$, one can find a sufficiently small compact neighbourhood $C_{i}$ of $p_{i}$ in $N$ such that (3) with $\gamma \in U_{i}\left(U_{i}\right.$ a small neighbourhood of 0 in $\left.\mathcal{F}(N)\right)$ has a $T$-periodic solution whose image is contained in $C_{i}$. This finishes the proof.

As we already remarked, in the case when $N$ is compact the only possible admissible integer is $s=|\chi(N)|$. Indeed, in this case, $\mathfrak{X}^{r}(N)=\mathfrak{X}^{r,|\chi(N)|}(N)$, and the fine topology coincides with the $C^{r}$ uniform. Hence we have:

Corollary 3.5. When $N$ is compact, $\mathfrak{X}_{T}^{r,|\chi(N)|}(N), r \geq 0$, is open and dense in $\mathfrak{X}^{r}(N)$ (with the uniform $C^{r}$ topology).

We stay with the case $N$ compact and, as in [6], restrict our attention to a particular class of first-order systems (3) whose leading term $\varphi$ is a gradient of some $C^{r}(r \geq 1)$ function $G: N \longrightarrow \mathbb{R}$, i.e.:

$$
\dot{x}=\operatorname{grad} G(x)+\gamma(t, x) .
$$

Denote by $g_{T}^{r}(N)$ the subspace of $C^{r}(N, \mathbb{R})$ of all the functions $G$ having the property that there exists a neighbourhood $U$ of 0 in $\mathcal{F}(N)$ such that (5) has at least

$$
b(N)=\sum_{i=0}^{n} b_{i}(N)
$$

geometrically distinct $T$-periodic solutions for any $\gamma \in U$. Here $b_{i}(N)$ denotes the $i$-th Betti number of $N$.

In view of the proof of Lemma 3.3 and Theorem 5.5 in [6], one gets:

Theorem 3.6. $g_{T}^{r}(N)(r \geq 1)$ is open and dense in $C^{r}(N, \mathbb{R})$.

Since $b(N)$ is greater than or equal to the Euler-Poincaré characteristic

$$
\chi(N)=\sum_{i=0}^{n}(-1)^{i} b_{i}(N),
$$

the above theorem gives a stronger result than Theorem 3.4 applied to Equation (5). For instance, if $N$ is the two-dimensional torus $\mathbf{T}^{2}$, one has $b\left(\mathbf{T}^{2}\right)=4$, whereas $\chi\left(\mathbf{T}^{2}\right)=0$. 


\section{Applications to second-order equations}

In this section we study the genericity of the multiplicity results for second-order ODEs on (not necessarily compact) boundaryless differentiable manifolds. We shall define the second-order analogues, $f_{T}^{r, s}(M)$ and $f^{r, s}(M)$, of the spaces $\mathfrak{X}_{T}^{r, s}$ and $\mathfrak{X}^{r, s}$ considered in the previous section, and show that the former is open and dense in the latter one. This result will, in particular, yield a generalization of the main result of [6] (Corollary 4.5).

In what follows, we take $N=T M \subset \mathbb{R}^{l}$ with $l=2 k, k$ being the dimension of the ambient space for $M$. We will say that $s \in \mathbb{N} \cup\{0\}$ is second-order admissible if there exists $h: T M \rightarrow \mathbb{R}^{k}$ tangent to $M$ such that $\left|\operatorname{deg}\left(\left.h\right|_{M}, M\right)\right|=s$. As in the previous section, when $M$ is compact, the only possible second-order admissible integer is $|\chi(M)|$.

Define

$$
s^{r}(M)=\left\{h \in C^{r}(T M): h(p, v) \in T_{p} M, \text { for any } p \in M, v \in T_{p} M\right\}
$$

and, for a second-order admissible integer $s$, let

$$
f^{r, s}(M)=\left\{h \in \mathcal{f}^{r}(M):\left.h\right|_{M} \in \mathfrak{X}^{s, r}(M)\right\} .
$$

Recall that, given $h: T M \rightarrow \mathbb{R}^{k}$ tangent to $M, \widehat{h}: T M \longrightarrow \mathbb{R}^{2 k}$ denotes the second-order vector field associated to $h$. Let $\theta: f^{r}(M) \longrightarrow \mathfrak{X}^{r}(T M)$ be the mapping that takes $h$ to $\widehat{h}$. Clearly $\theta$ is injective. Put $y^{r}(M)=\theta\left(f^{r}(M)\right)$, and define $y^{r, s}(M)=\mathcal{Y}^{r}(M) \cap \mathfrak{X}^{r, s}(T M)$. By Lemma 3.2 in [4], $y^{r, s}(M)=\theta\left(f^{r, s}(M)\right)$.

Since $\mathcal{Y}^{r}(M)$ and $\mathcal{Y}^{r, s}(M)$ are contained in $\mathfrak{X}^{r}(T M)$, they naturally inherit the topology from $\mathfrak{X}^{r}(T M)$. Moreover, considering $f^{r}(M)$ as a topological subspace of $C^{r}\left(T M, \mathbb{R}^{k}\right)$ endowed with the fine (Whitney) topology, one can check that $f^{r}(M)$ and $f^{r, s}(M)$ are, respectively, homeomorphic to $y^{r}(M)$ and $y^{r, s}(M)$.

Lemma 4.1. Any open subset of $y^{r, s}(M), r \geq 1$, contains a vector field $\omega$ whose zeros are non-degenerate. Moreover, $\# \omega^{-1}(0) \geq s$.

Proof. It is enough to prove the assertion for the basic neighbourhoods of the topology on $\mathcal{Y}^{r, s}(M)$. Therefore, given any $\varphi$ in $\mathcal{y}^{r, s}(M)$, consider any of its basic neighbourhood $U$. By the definition of the topology on $y^{r, s}(M), U$ is given by

$$
U=\mathcal{N}^{r}(\varphi, \Psi, \mathcal{K}, E) \cap \mathcal{Y}^{r, s}(M),
$$

for some fixed families $\Psi=\left\{\psi_{i}\right\}_{i \in \Lambda}, \mathcal{K}=\left\{K_{i}\right\}_{i \in \Lambda}$ and $E=\left\{\varepsilon_{i}\right\}_{i \in \Lambda}$.

We shall prove that $U$ contains a vector field $\omega$ as in the assertion. This, by the arbitrariness of the choices of $\varphi$ and $U$ will prove the lemma.

By the definition of $y^{r, s}(M)$, there exists $h_{0}: T M \longrightarrow \mathbb{R}^{k}, C^{r}$, tangent to $M$, such that $\left.h_{0}\right|_{M} \in \mathfrak{X}^{r, s}(M)$ with the property that $\varphi=\widehat{h_{0}}$. Define

$$
\widetilde{\Psi}=\left\{\widetilde{\psi}_{i}, \widetilde{U}_{i}\right\}_{i \in \Lambda}, \quad \widetilde{\mathcal{K}}=\left\{\widetilde{K}_{i}\right\}_{i \in \Lambda}, \quad \text { and } \quad \widetilde{E}=\left\{\widetilde{\varepsilon}_{i}\right\}_{i \in \Lambda},
$$


where $\widetilde{U}_{i}=U_{i} \cap M, \widetilde{\psi}_{i}=\psi_{i} \widetilde{U}_{i}, \widetilde{K}_{i}=K_{i} \cap M$, and $\widetilde{\varepsilon}_{i}=\varepsilon_{i} / 2$. Let

$$
\widetilde{U}=\mathcal{N}^{r}\left(\left.h_{0}\right|_{M}, \widetilde{\Psi}, \widetilde{\mathcal{K}}, \widetilde{E}\right) \cap \mathfrak{X}^{r, s}(M) .
$$

By Proposition 3.2, there exists $h_{1} \in \widetilde{U}$ such that all its zeros are non-degenerate and $\# h_{1}^{-1}(0) \geq s$.

Let $\sigma: T M \longrightarrow[0,1]$ be a smooth function such that $\left.\sigma\right|_{M}=1$. If the support of $\sigma$ is a small enough neighbourhood of $M$, then one has that the function $h: T M \longrightarrow \mathbb{R}^{k}$,

$$
h(p, v)=\sigma(p, v) h_{1}(p)+(1-\sigma(p, v)) h_{0}(p, v),
$$

satisfies $\widehat{h} \in \mathcal{N}^{r}(\varphi, \Psi, \mathcal{K}, E)$. It is easy to check that $h$ is $C^{r}$, tangent to $M$ and $\left.h\right|_{M}=h_{1} \in \mathfrak{X}^{r, s}(M)$.

Let $\omega=\widehat{h}$. Then $\omega \in U$ and $\omega^{-1}(0)=h_{1}^{-1}(0)$, consequently $\# \omega^{-1}(0) \geq s$. Take $p \in \omega^{-1}(0)$.

Since

$$
T_{(p, 0)} T M=T_{p} M \times T_{p} M,
$$

the linear operator $\omega^{\prime}(p, 0): T_{(p, 0)} T M \longrightarrow T_{(p, 0)} T M$ is represented by the block matrix:

$$
\left(\begin{array}{cc}
0 & I \\
D_{1} h(p, 0) & D_{2} h(p, 0)
\end{array}\right)=\left(\begin{array}{cc}
0 & I \\
D_{1} h_{1}(p, 0) & D_{2} h(p, 0)
\end{array}\right),
$$

where $I$ is the identity on $T_{p} M$. Therefore

$$
\operatorname{det} \omega^{\prime}(p, 0)=(-1)^{m} \operatorname{det} h_{1}^{\prime}(p),
$$

where $m$ is the dimension of $M$. Consequently, all zeros of $\omega$ are non-degenerate.

We now establish a technical lemma that, in the framework of second-order differential equations, plays the same role as Lemma 3.3 in the previous section.

Lemma 4.2. Assume that $\varphi \in \mathcal{Y}^{r, s}(M), r \geq 1$, has $\sigma$ non-degenerate zeros $z_{1}, \ldots, z_{\sigma}$. Then, given a neighbourhood $U$ of $\varphi$ in $y^{r, s}(M)$, there exists $\omega \in U$ such that $z_{1}, \ldots, z_{\sigma}$ are second-order non-T-resonant zeros of $\omega$.

Proof. Since $\varphi$ is in $\mathcal{Y}^{r, s}(M)$, we have $\varphi=\widehat{h}_{0}$ for some $h_{0}: T M \longrightarrow \mathbb{R}^{k}$ of $C^{r}$ class, tangent to $M$ and such that $\left.h_{0}\right|_{M} \in \mathfrak{X}^{r, s}(M)$, and with the property that the points $p_{1}, \ldots, p_{\sigma}$, defined by $\left(p_{i}, 0\right)=z_{i}, i=1, \ldots, \sigma$, are non-degenerate zeros of $\left.h_{0}\right|_{M}$.

Exactly as in the proof of Lemma 4.1, but using Lemma 3.3 instead of Proposition 3.2 , we get a vector field $\omega=\widehat{h} \in U$ with $p_{1}, \ldots, p_{\sigma}$ being (first-order) non- $T$-resonant zeros of $\left.h\right|_{M}$. Thus $z_{1}, \ldots, z_{\sigma}$ are second-order non- $T$-resonant zeros of $\omega$ and the result follows. 
Analogously to the space $\mathfrak{X}_{T}^{r, s}(N)$ introduced in Sect. 3, we define the space $f_{T}^{r, s}(M) \subset f^{r, s}(M)$, made out of those $h: T M \longrightarrow \mathbb{R}^{k}$, tangent to $M$, for which Equation (2) admits at least $s$ geometrically distinct $T$-periodic solutions whenever $f$ belongs to an appropriate open neighbourhood of 0 in $\mathcal{E}(M)$. We also put $y_{T}^{r, s}(M)=\theta\left(s_{T}^{r, s}(M)\right)$.

We summarize the relations between the spaces introduced above in the following table:

\begin{tabular}{|l|l|l|}
\hline \multicolumn{3}{|c|}{ Relations induced by the correspondence $h \stackrel{\theta}{\longmapsto} \widehat{h}$} \\
\hline \hline The space: & \multicolumn{1}{|c|}{ consists of: } & corresponds to: \\
\hline$f^{r}(M)$ & $\begin{array}{l}\text { any } h \in C^{r}\left(T M, \mathbb{R}^{k}\right) \text { s.t. } \\
h(p, v) \in T_{p} M \text { for any } \\
(p, v) \in T M\end{array}$ & $\mathcal{Y}^{r}(M) \subset \mathfrak{X}^{r}(T M)$ \\
\hline$f^{r, s}(M)$ & $\begin{array}{l}\text { any } h \in 8^{r}(M) \quad \text { s.t. } \\
\left|\operatorname{deg}\left(\left.h\right|_{M}, M\right)\right|=s\end{array}$ & $\mathcal{Y}^{r, s}(M) \subset \mathfrak{X}^{r, s}(T M)$ \\
\hline$f_{T}^{r, s}(M)$ & $\begin{array}{l}\text { any } h \in \delta^{r, s}(M) \text { s.t. (2) } \\
\text { has } s T \text {-periodic solutions } \\
\text { whenever } f \text { belongs to a }\end{array}$ & $\mathcal{Y}_{T}^{r, s}(M) \subset \mathfrak{X}_{T}^{r, s}(T M)$ \\
\hline
\end{tabular}

In view of Lemmas 4.1 and 4.2 above and of Theorem 4.1 in [6], arguing as in the proof of Theorem 3.4, we get the following result:

Lemma 4.3. The set $y_{T}^{r, s}(M), r \geq 0$, is open and dense in $y^{r, s}(M)$.

We are now ready to state the second-order analogue of Theorem 3.4, which, roughly speaking, asserts that for 'almost any' $h \in \delta^{r, s}(M)$ Equation (2) admits at least $s$ geometrically distinct $T$-periodic solutions for any 'small' $T$-periodic perturbation $f$.

Theorem 4.4. The set $f_{T}^{r, s}(M), r \geq 0$, is open and dense in $f^{r, s}(M)$.

Proof. It follows immediately from Lemma 4.3 and from the homeomorphism of $f^{r}(M)$ and of $f^{r, s}(M)$ with $y^{r}(M)$ and $y^{r, s}(M)$, respectively.

The following corollary is a generalization of Theorem 5.1 in [6], where the function $g$ in (2) was assumed to depend only on the position $p$ (and not on the speed $v)$.

Corollary 4.5. When $M$ is compact, $f_{T}^{r,|\chi(M)|}(M), r \geq 0$, is open and dense in $8^{r}(M)$.

\section{References}

1. Furi, M.: Second order differential equations on manifolds and forced oscillations. Proc. Conference on Topological Methods in Differential Equations and Inclusions, Université de Montréal, 1994, NATO ASI series C. Dordrecht: Kluwer Acad. Publ. 
2. Furi, M., Pera, M.P.: A continuation principle for periodic solutions of forced motion equations on manifolds and applications to bifurcation theory. Pacific J. of Math. 160, 219-244 (1993)

3. Furi, M., Spadini, M.: Multiplicity of forced oscillations for the spherical pendulum. Topological Methods in Nonlinear Analysis 11, 147-157 (1998)

4. Furi, M., Spadini, M.: Branches of forced oscillations for periodically perturbed autonomous second order ODE's on manifolds. J. of Differential Equations 154, 96-106 (1999)

5. Hirsch, M.W.: Differential Topology. Graduate Texts in Math. 33. New York: Springer 1976

6. Lewicka, M., Spadini, M.: On the genericity of the multiplicity results for forced oscillations on compact manifolds. NoDEA Nonlin. Differ. Equ. Appl. 6, 357-369 (1999)

7. Milnor, J.W.: Morse Theory. Annals of Math. Studies 51. Princeton: Princeton Univ. Press 1963

8. Milnor, J.W.: Topology from the differentiable viewpoint. Charlottesville: The University Press of Virginia 1965

9. Palis jr., J., de Melo, W.: Geometric theory of dynamical systems. New York: Springer 1982

10. Spadini, M.: Harmonic solutions of periodic Carathéodory perturbations of autonomous ODE's on manifolds. Nonlin. Analysis TMA 41A, 477-487 (2000) 